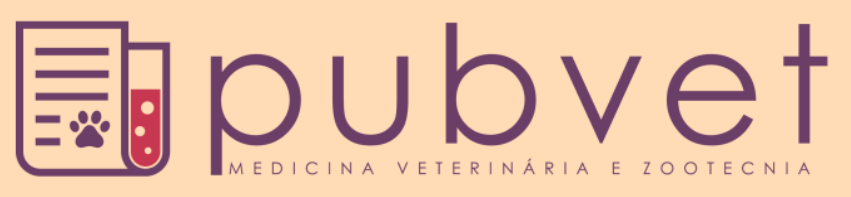

HTTP://DX.DOI.ORG/10.22256/PUBVET.V11N10.996-1001

\title{
Comparação de alfa-2-adrenérgicos na anestesia epidural sacrococcigea de gatas submetidas à ovariosalpingohisterectomia
}

\author{
João Araújo Melo Neto ${ }^{1 *}$, Júlio Cézar dos Santos Nascimento², Lucilo Bioni da Fonsêca \\ Filho $^{3}$, Mauro de Araújo Pena Forte Junior ${ }^{4}$, Kleyton Domingos de Melo ${ }^{4}$, Cláudio \\ Roberto Soares Espíndola ${ }^{5}$, Ana Paula Monteiro Tenório ${ }^{6}$

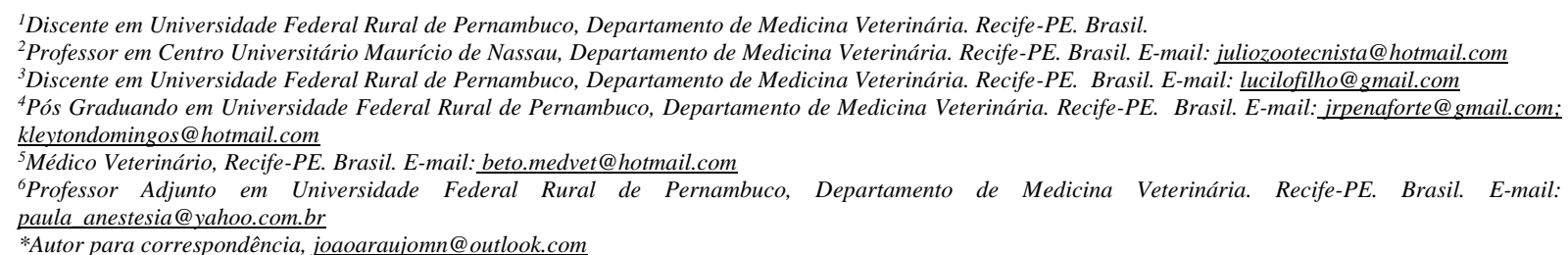

RESUMO. A pesquisa foi desenvolvida no Hospital Veterinário, do Departamento de Medicina Veterinária da Universidade Federal Rural de Pernambuco, onde foram utilizadas 15 gatas hígidas, com idade entre um e três anos, com peso que variou entre três e quatro quilos distribuídas em três grupos e submetidas à ovary salpingohisterectomia. Os animais foram pré-medicados com acepromazina $2 \%, 0,05 \mathrm{mg} / \mathrm{kg}$. O cloridrato de cetamina 50 $\mathrm{mg} / \mathrm{ml}, 10 \mathrm{mg} / \mathrm{kg}$ e diazepam $5 \mathrm{mg} / \mathrm{ml}, 0,5 \mathrm{mg} / \mathrm{kg}$, foram utilizados para a indução anestésica e a manutenção foi feita utilizando-se o cloridrato de cetamina na dose de 4 $\mathrm{mg} / \mathrm{kg}$. Em seguida, foram submetidas a anestesia epidural sacrococcígea utilizando-se apenas clonidina na dose de $20 \mu \mathrm{g} / \mathrm{kg}$ ou xilazina na dose $2 \mathrm{mg} / \mathrm{kg}$ ou solução salina (placebo) $1 \mathrm{ml} / 3,5 \mathrm{~kg}$. Os animais foram avaliados quanto aos parâmetros hemodinâmicos, respiratórios e temperatura corporal, além da avaliação do potencial analgésico e intensidade do bloqueio alcançado a partir da mensuração de glicose, lactato e cortisol, desde o período pré-anestésico até o término da cirurgia

Palavras chave: anestesiologia, gatos, veterinária

\section{Comparison of alpha-2-adrenoreceptors in the epidural anesthesia sacrococcigea of cats undergoing ovariohysterectomy}

ABSTRACT. The research was carried out at the Veterinary Hospital, the Department of Veterinary Medicine of Rural Federal University of Pernambuco, where they were used 15 cats undergoing ovariosalpingohisterectomy, participated and aged between one and three years, with weight that ranged between three and four pounds, divided into three groups, submitted to ovariosalpingohisterectomy. The animals were pre-medicated with acepromazine $2 \%, 0.05 \mathrm{mg} / \mathrm{kg}$. The Ketamine hydrochloride $50 \mathrm{mg} / \mathrm{ml}, 10 \mathrm{mg} / \mathrm{kg}$ and diazepam $5 \mathrm{mg} / \mathrm{ml}, 0.5 \mathrm{mg} / \mathrm{kg}$, were used for anesthetic induction and maintenance was performed using the ketamine hydrochloride at a dose of $4 \mathrm{mg} / \mathrm{kg}$. Then, they were subjected to epidural anesthesia the sacrococcygeal using only clonidine in dose of 20 $\mu \mathrm{g} / \mathrm{kg}$ or xylazine at the dose $2 \mathrm{mg} / \mathrm{kg}$ or saline (placebo) $1 \mathrm{ml} / 3.5-\mathrm{kg}$. The animals were assessed in relation to hemodynamic parameters, respiratory and body temperature, in addition to the evaluation of the analgesic potential and intensity was reached from the measurement glucose, lactate and cortisol levels, since the pre-anesthetic until the end of the surgery. 
Keywords: anesthesiology, cats, veterinary

\section{Comparación de alfa-2-adrenoreceptors en la anestesia epidural de gatas sometidas a ovariosalpingohisterectomía}

RESUMEN. La investigación fue desarrollada en el Hospital Veterinario, del Departamento de Medicina Veterinaria de la Universidad Federal Rural de Pernambuco, donde se utilizaron 15 gatas sanas, con edad entre uno y tres años, con peso que varía entre tres y cuatro kilogramos, distribuidas en tres grupos, sometidas a la ovariosalpingohisterectomía. Los animales fueron premedicados con acepromazina $2 \%$, $0,05 \mathrm{mg} / \mathrm{kg}$. El clorhidrato de cetamina $50 \mathrm{mg} / \mathrm{ml}, 10 \mathrm{mg} / \mathrm{kg}$ y diazepam $5 \mathrm{mg} / \mathrm{ml}, 0,5$ $\mathrm{mg} / \mathrm{kg}$, fueron utilizados para la inducción anestésica y el mantenimiento se efectuó utilizando el clorhidrato de cetamina a dosis de $4 \mathrm{mg} / \mathrm{Kg}$. A continuación, se sometió a la anestesia epidural sacrococcígea utilizando sólo clonidina en la dosis de $20 \mu \mathrm{g} / \mathrm{kg}$ o xilazina en la dosis $2 \mathrm{mg} / \mathrm{kg}$ o solución salina (placebo) $1 \mathrm{ml} / 3,5 \mathrm{~kg}$. Los animales fueron evaluados en cuanto a los parámetros hemodinámicos, respiratorios y temperatura corporal, además de la evaluación del potencial analgésico e intensidad del bloqueo alcanzado a partir de la medición de glucosa, lactato y cortisol, desde el período pre anestésico hasta el término de la cirugía.

Palabras clave: anestesiología, gatos, veterinaria

\section{Introdução}

Partindo da afirmação que intervenções cirúrgicas desencadeiam mecanismos dolorosos, utiliza-se como ferramenta a anestesia com finalidade de prevenir e reduzir a dor trans e pósoperatória (Muir et al., 1992). Um procedimento anestésico eficaz envolve diferentes técnicas e mecanismos de ação, promovidos pela atuação de mais de um fármaco além do utilizado na anestesia geral, uma vez que esta não possui ação analgésica (Massone, 1999).

Dentre as técnicas anestésicas locais está a anestesia epidural, que consiste no bloqueio regional com injeção do anestésico no espaço entre a dura-máter e o canal vertebral, podendo ser na região torácica, lombar ou caudal (Collins, 1976), agindo com efeito de paralisia temporária das regiões inervadas pela área na qual foi administrado o fármaco (Fantoni et al., 2002).

Sua aplicabilidade é ampla, e tem sido utilizada frequentemente, pela possibilidade da redução da dose de fármacos, quando comparado à administração por outras vias. Possui ainda, menores efeitos adversos, promovem analgesia trans e pós-operatória (Polydoro et al., 2006) e permitem a diminuição da resposta ao estresse pós-cirúrgico (Weissman, 1990).

A técnica é indicada em casos de pacientes de risco, cardiopatas, patologias do trato respiratório, distúrbios metabólicos que impossibilitem a utilização de anestesia geral (Collins, 1976). Cirurgias nos membros pélvicos, na coxa, região anal, perianal e caudal constituem o grupo de principais indicações para a realização da anestesia epidural, embora ultimamente tenha se usado esta técnica em cirurgias abdominais e torácicas (Fantoni et al., 2002).

Segundo Polydoro et al. (2006), a utilização desta técnica é eficaz para realização de procedimentos caudais ao diafragma, entretanto seu efeito mostrou-se superior para procedimentos de membros posteriores, região perineal e pélvica. Há contraindicação para pacientes no estado de choque ou hemorragia, com defeitos de coagulação ou inflamação local (Collins, 1976).

Os anestésicos locais são substâncias empregadas para o bloqueio local, pois são capazes de bloquear o canal de sódio impedindo a despolarização da membrana (Massone, 1999), bloqueando a sensibilização central à dor, exercendo efeito regulatório negativo da informação nociceptiva produzindo analgesia (Bassanezi and Oliveira Filho, 2006). Quando administrados pela via epidural produzem bloqueio indiscriminado sensorial, simpático e motor. Seus efeitos adversos como a toxicidade, estão ligados a altas concentrações ou aplicações inapropriadas (Nelson and Couto, 2015).

A xilazina é um. $\alpha 2$ - agonista e tem sua ação nos receptores $\alpha 2$, mas também chega a se ligar nos receptores $\alpha 1$. Com a ativação dos receptores $\alpha 2$ há uma redução na formação do AMPc, que é um importante regulador do funcionamento celular. Também com a ativação da proteína $\mathrm{G}$ a 
canais iônicos de potássio há uma redução na excitabilidade neuronal e certo nível de sedação é observado após o uso da xilazina (Spinosa et al., 1999).

Atualmente, tem-se avaliado outros fármacos, entre eles os pertencentes à família dos $\alpha 2$ agonistas (LeBlanc and Caron, 1990, Valadão et al., 2002a) como a clonidina, por exemplo, uma vez que possuem a capacidade de produzir maior seletividade sensorial e maior período de ação, e seus efeitos analgésicos estão relacionados com a redução de norepinefrina periférica, inibição da transmissão nervosa nociceptiva no corno dorsal da medula espinhal por mecanismos póssinápticos e inibição direta dos neurônios simpáticos espinhais pré-ganglionares (Valadão et al., 2002b).

Os $\alpha$-2 agonistas são utilizados na medicina veterinária principalmente como medicação préanestésica, visto que promovem sedação, miorrelaxamento e analgesia dose-dependente. Produzem depressão do sistema nervoso central. Embora não possuam efeito analgésico de primeira linha, como os agentes opióides ou antiinflamatórios não esteróides, os $\alpha$-2 agonistas são utilizados como analgésicos adjuvantes (Massone, 1999). São empregados também como agentes sedativos e pré-anestésicos principalmente por propiciar a redução da concentração alveolar mínima (CAM) de anestésicos inalatórios e na dose de outros fármacos (Maze and Tranquilli, 1991). Quando administrado pela via epidural, apresenta efeito antinociceptivo como resultado do estímulo de $\alpha-2$ adrenoreceptores na medula espinhal. A ligação aos receptores provoca a liberação de noradrenalina, hiperpolarização dos neurônios do corno dorsal e inibição da substância $\mathrm{P}$, produzindo analgesia (Lemke, 2004).

Em função da similaridade da estrutura química dos $\alpha-2$ agonistas com a da lidocaína, os mesmos exercem excelente efeito analgésico visceral (Greene, 2004).

A clonidina é um agonista $\alpha-2$ parcial adrenérgico pré-sináptico com propriedades ansiolíticas e hipnóticas. Quando empregada em anestesia loco-regional é capaz de promover analgesia mediante ação em um sítio espinhal, sem induzir a depressão respiratória, náusea ou vômito, entretanto, produz bradicardia e hipotensão (Eisenach et al., 1993, Gasparini et al., 2007). Mecanismos sobre os receptores adrenérgicos sinápticos, mediante atividade da clonidina, aumentam a duração do bloqueio sensitivo e motor (Rochette et al., 2004, Strebel et al., 2004). Portanto, pode ser utilizada como fármaco adjuvante no controle da dor pós-operatória pela via espinhal (Förster and Rosenberg, 2004, Schechtmann et al., 2004).

Uma vez que a mensuração, reconhecimento e tratamento da dor têm sido cada vez mais estudados na medicina veterinária, tornou-se frequente uso da analgesia preventiva evitando a sensibilização central em função da liberação de mediadores inflamatórios liberados após lesão tecidual e seus efeitos deletérios (Maddison et al., 2011). Concomitantemente, o conhecimento acerca dos fármacos e suas propriedades, permitem melhor aplicabilidade do uso destes no controle da dor, proporcionando melhor bem-estar e recuperação do paciente (Fantoni et al., 2002).

Segundo a Associação Internacional para o Estudo da Dor (IASP), define-se a dor como uma experiência emocional e sensorial desagradável associada a dano tecidual. Por isso as utilidades de muitas medidas da dor, como, por exemplo, as escalas e os questionários multidimensionais, resultam parcialmente do reconhecimento e da avaliação independente desses diferentes componentes ou dimensões da dor (Silva and Ribeiro-Filho, 2011). Avanços nos estudos da neurofisiologistas têm esclarecido o mecanismo de estresse nos animais. Como indicadores sanguíneos de estresse mais eficientes temos o cortisol, lactato, e a glicose (Holst, 1999, Guyton and Hall, 2006). O aumento dos valores séricos de hormônios glicocorticoides, entre eles o cortisol, ocorre pela ativação do sistema endócrino em situações de estresse (Barra Filho et al., 2002), bem como a queda no aporte tecidual de oxigênio que ocorre em consequência do estímulo de lesão tecidual promove o catabolismo anaeróbico da glicose, que tem como produto final o lactato, consequentemente acidose. Portanto, a mensuração do lactato sérico torna-se um aliado para detecção de situações como esta (Allen and Holm, 2008).

\section{Material e Métodos}

O presente estudo foi enviado à Comissão de Ética no Uso de Animais da Universidade Federal Rural de Pernambuco. Para utilização de cadelas que era o projeto inicial, recebeu a autorização número 046/2013.

Para a realização do presente estudo foram utilizadas 15 gatas submetidas à ovariosalpingohisterectomia (OSH), hígidas e com idade entre 
um e três anos, com peso entre três e quatro quilos, oriundas da comunidade carente adjacente a Universidade e também animais submetidos aos cuidados do gatil do Departamento de Medicina Veterinária da Universidade Federal Rural de Pernambuco, com prévia autorização do respectivo tutor.

Os animais foram classificados em três grupos, de cinco animais cada, de acordo com a solução empregada na anestesia epidural, sendo estes:

Grupo 1: Clonidina (GC), na dose de $20 \mu \mathrm{g} / \mathrm{kg}$ pela via epidural sacrococcígea;

Grupo 2: Xilazina (GX), na dose de $2 \mathrm{mg} / \mathrm{kg}$ por via epidural sacroccígea;

Grupo 3: Solução Salina (GS), no volume de 1 $\mathrm{ml} / 3,5 \mathrm{~kg}$ por via epidural sacrococcígea.

Em todos os protocolos foi adotado um volume máximo final de $1 \mathrm{ml} / 3,5 \mathrm{~kg}$.

A avaliação dos pacientes foi feita previamente mediante avaliação clínica, bem como testes para determinação do tempo de coagulação e sangria com a finalidade de obter informações que corroborem com o pré-requisito de higidez.

Todos os animais foram pré-medicados com acepromazina $2 \%, 0,05 \mathrm{mg} / \mathrm{kg}$. O cloridrato de cetamina $50 \mathrm{mg} / \mathrm{ml}, 10 \mathrm{mg} / \mathrm{kg}$ e diazepam 5 $\mathrm{mg} / \mathrm{ml}, 0,5 \mathrm{mg} / \mathrm{kg}$ foram utilizados para a indução anestésica e a manutenção foi feita utilizando-se o cloridrato de cetamina na dose de $4 \mathrm{mg} / \mathrm{kg}$. Posteriormente será realizado o bloqueio epidural, após tricotomia e antissepsia do local com álcool 70 e clorexidina.

Foram registrados os parâmetros fisiológicos de cada paciente, como a frequência respiratória (FR), frequência cardíaca (FC), pulso (P) temperatura $\left(\mathrm{t}^{\circ}\right)$, em quatro momentos específicos: M1 - parâmetros basais; M2 - após 15minutos da MPA; M3 - após instalação da epidural; M4 - após o término da cirurgia.

Os procedimentos cirúrgicos foram realizados por um mesmo cirurgião, devidamente treinado, que atribuiu nota em relação à facilidade ou não de tração dos pedículos ovarianos, de acordo com o escore estabelecido no Quadro 1.

Foi efetuada coleta de $3 \mathrm{ml}$ de sangue venoso, por punção da veia jugular, sem anticoagulante, nos momentos M1, M3 e M4 para mensuração de glicose através de glicosímetro Accu-Chek Advantage da Roche $^{\circledR}$. Para a mensuração do cortisol o sangue foi sorado naturalmente e centrifugado por cinco minutos, numa rotação de
3500RPM, separado e identificado em Ependorff. As amostras estão congeladas a $-18^{\circ}$ Celsius para posterior mensuração do cortisol sérico pela técnica de Eletroquioluminescência em equipamento Cobas E411 da Roche®.

Quadro 1. Escore de avaliação cirúrgica da tração do pedículo ovariano.

\begin{tabular}{|c|l|}
\hline Score & \multicolumn{1}{|c|}{ Critério } \\
\hline 1 & $\begin{array}{l}\text { Total resistência à tração dos pedículos } \\
\text { ovarianos }\end{array}$ \\
\hline 2 & $\begin{array}{l}\text { Alguma resistência à tração dos pedículos } \\
\text { ovarianos }\end{array}$ \\
\hline 3 & $\begin{array}{l}\text { Nenhuma resistência à tração dos } \\
\text { pedículos ovarianos. }\end{array}$ \\
\hline
\end{tabular}

\section{Resultados e Discussão}

O tempo de instalação da peridural no grupo 1 (GC) foi entre 3 e 10 minutos para que pudesse ser evidenciado relaxamento do esfíncter anal, perda de reflexos nos membros inferiores e cauda. Já no grupo 2 (GS) não houve nenhum sinal de relaxamento do esfíncter anal nem perda sensorial, mesmo sendo obedecida a espera mínima de 30 minutos para a instalação da peridural. No grupo 3 (GX) o intervalo de tempo para que fosse observado qualquer relaxamento de esfíncter anal ou perda sensorial foi o mesmo do grupo 1 (GC) mas foi evidenciado que o relaxamento não tinha a mesma intensidade que o do grupo 1 (GC). O tempo de cirurgia variou entre 30 e 45 minutos, sendo perguntado ao cirurgião escores parciais, onde ficou evidenciado que animais do grupo 2 (GS) e grupo 3 (GX) tinham praticamente o mesmo miorrelaxamento trans-cirúrgico, sendo atribuída a eles escore 2 do cirurgião em todo trans e pós-operatório, diferente do grupo 1 (GC) que recebeu escore $3 \mathrm{em}$ todos os momentos. $\mathrm{O}$ número de resgates anestésicos também variou entre os grupos, no grupo 1 (GC) o número de resgates não passou de 3 , já no grupo $2(\mathrm{GS}) \mathrm{o}$ número de resgates chegou a $5 \mathrm{em}$ dois animais e foi necessário o uso de resgate analgésico em 4 dos 5 animais operados. No grupo 3 (GX) o número de resgates anestésicos não passou de 4 sendo necessário 2 apenas em um animal dos 5 que compunham este grupo.

Como podemos observar na Tabela 1 a média da frequência cardíaca no grupo 1 (GC) foi mais baixa do que os demais grupos, igualmente na Tabela 2 que demonstra um menor número de movimentos respiratórios no grupo 1 (GC) do que nos demais grupos. Esses fatores levam a crer que os animais do grupo 1 (GC) apresentaram boa 
analgesia, mais efetiva do que os demais grupos, uma vez que os parâmetros desse grupo não apresentaram alterações fisiológicas dignas de nota. Em contrapartida na Tabela 3 evidencia-se a média da glicose, onde se observa que o grupo 1 (GC) foi o que apresentou uma maior média no pós-operatório, podendo caracterizar dor, mas isto pode ocorrer por que os animais do grupo 1 (GC) foram submetidos a menos resgates anestésicos.

Tabela 1. Médias da frequência cardíaca de gatas anestesiadas com alfa-2 receptores por via peridural submetidas à ovariosalpingohisterectomia. Recife, 2014.

\begin{tabular}{lllll}
\hline Grupos & Basal & $\begin{array}{l}\text { Pós- } \\
\text { MPA }\end{array}$ & $\begin{array}{l}\text { Pós- } \\
\text { epidur } \\
\text { al }\end{array}$ & $\begin{array}{l}\text { Pós- } \\
\text { cirurgi } \\
\text { a }\end{array}$ \\
\hline Clonidina (GC) & 194,0 & 159,0 & 140,6 & 164,6 \\
Xilazina (GX) & 195,6 & 173,6 & 148,2 & 179,6 \\
Solução Salina (GS) & 212,8 & 205,0 & 164,6 & 171,2 \\
\hline
\end{tabular}

Tabela 2. Médias da frequência respiratória de gatas anestesiadas com alfa-2 receptores por via peridural submetidas à ovariosalpingohisterectomia. Recife, 2014.

\begin{tabular}{lcccc}
\hline Grupos & Basal & $\begin{array}{c}\text { Pós- } \\
\text { MPA }\end{array}$ & $\begin{array}{l}\text { Pós- } \\
\text { epidural }\end{array}$ & $\begin{array}{l}\text { Pós- } \\
\text { cirurgia }\end{array}$ \\
\hline Clonidina (GC) & 38,0 & 43,6 & 30,8 & 27,6 \\
Xilazina (GX) & 56,8 & 43,2 & 38,0 & 33,6 \\
Solução Salina (GS) & 46,6 & 37,2 & 29,6 & 31,2 \\
\hline
\end{tabular}

Tabela 3. Médias da glicose de gatas anestesiadas com alfa2 receptores por via peridural submetidas à ovariosalpingohisterectomia. Recife, 2014.

\begin{tabular}{lccc}
\hline Grupos & Basal & $\begin{array}{c}\text { Pós } \\
\text { epidural }\end{array}$ & $\begin{array}{c}\text { Pós } \\
\text { cirurgia }\end{array}$ \\
\hline Clonidina (GC) & 88,8 & 127,6 & 183,4 \\
Xilazina (GX) & 93,6 & 121,4 & 119,2 \\
Solução Salina (GS) & 88,8 & 82,0 & 89,8 \\
\hline
\end{tabular}

Por fim a anestesia peridural sacrococcígea no grupo 1 (GC) apresentou-se mais eficaz causando melhor miorrelaxamento e analgesia trans e pósoperatória, ficou também evidenciado pelo cirurgião que a exposição tanto dos pedículos ovarianos quanto do coto uterino foi facilitada quando se utilizou a clonidina por via peridural (Tabela 4).

Tabela 4. Médias do escore do cirurgião para as gatas anestesiadas com alfa-2 receptores por via peridural submetidas à ovariosalpingohisterectomia. Recife, 2014.

\begin{tabular}{ll}
\hline Grupos & Basal \\
\hline Clonidina (GC) & 3,0 \\
Xilazina (GX) & 1,8 \\
Solução Salina (GS) & 1,6 \\
\hline
\end{tabular}

\section{Conclusão}

A frequência cardíaca e o número de movimentos respiratórios no grupo de animais anestesiados com Clonidina foram menores do que nos animais anestesiados com Xilazina e solução salina. Esses fatores mostram que os animais anestesiados com Clonidina apresentaram boa analgesia e mais efetiva do que nos animais dos demais grupos, uma vez que os parâmetros desse grupo não apresentaram alterações fisiológicas, significativas. Em contrapartida evidenciou-se maior média de glicose no pósoperatório, podendo caracterizar dor; mas isto também pode ter ocorrido por que os animais anestesiados com Clonidina foram submetidos a menor resgates anestésicos.

\section{Referências Bibliográficas}

Allen, S. E. \& Holm, J. L. 2008. Lactate: physiology and clinical utility. Journal of Veterinary Emergency and Critical Care, 18, 123-132.

Barra Filho, M. G., Ribeiro, L. C. S., Miranda, R. \& Teixeira, M. T. 2002. A redução dos níveis de cortisol sanguíneo através da técnica de relaxamento progressivo em nadadores. Revista Brasileira de Medicina do Esporte, 8, 139-143.

Bassanezi, B. S. B. \& Oliveira Filho, A. G. 2006. Analgesia pós-operatória. Revista do Colégio Brasileiro de Cirurgiões, 33, 116-122.

Collins, V. J. 1976. Principles of anesthesiology, Philadelphia, Pennsylvania.

Eisenach, J., Detweiler, D. \& Hood, D. 1993. Hemodynamic and analgesic actions of epidurally administered clonidine. Anesthesiology, 78, 277-287.

Fantoni, D. T., Mastrocinque, S., Fantoni, D. T. \& Cottopassi, S. R. 2002. Fisiopatologia e controle da dor. In: Fabntoni, D. T. (ed.) Anestesia em cães e gatos. Roca, São Paulo.

Förster, J. G. \& Rosenberg, P. H. 2004. Small dose of clonidine mixed with low-dose ropivacaine and fentanyl for epidural analgesia after total knee arthroplasty. British Journal of Anaesthesia, 93, 670-677.

Gasparini, S. S., Luna, S. P. L., Cassu, R. N., Uiechi, E. \& Crocci, A. J. 2007. Anestesia epidural com ropivacaína, lidocaína ou associação de lidocaína e xilazina em cães: efeitos cardiorrespiratório e analgésico. Ciência Rural, 41, 418-424. 
Greene, S. 2004. Segredos em anestesia veterinária e manejo da dor. Artmed, Porto Alegre.

Guyton, A. C. \& Hall, J. E. 2006. Tratado de fisiologia médica. Elsevier Brasil.

Holst, S. 1999. Assessment of stun-stick interval in relation to time of exposure in $\mathrm{CO} 2$ stunning of pigs. Animal welfare implications. international Workshop on stunning systems for Pigs and Animal Welfare. Billund, Denmark.

LeBlanc, P. H. \& Caron, J. P. 1990. Clinical use of epidural xylazine in the horse. Equine Veterinary Journal, 22, 180-181.

Lemke, K. A. 2004. Perioperative use of selective alpha-2 agonists and antagonists in small animals. The Canadian Veterinary Journal, 45, 475-480.

Maddison, J. E., Page, S. W. \& Church, D. B. 2011. Farmacologia clínica de pequenos animais. In: Philip, G. A. \& Thomas, A. F. (eds.) Medicamentos e reprodução. Elsevier Brasil, São Paulo.

Massone, F. 1999. Anestesiologia veterinária. Guanabara Koogan, Rio de Janeiro.

Maze, M. \& Tranquilli, W. 1991. Alpha-2 adrenoceptor agonistsDefining the role in clinical anesthesia. Anesthesiology: The Journal of the American Society of Anesthesiologists, 74, 581-605.

Muir, W. W., Hubbell, J. A. E., Skarda, R. T. \& Bednarski, M. R. 1992. Manual de anestesia veterinária. Acribia.

Nelson, R. W. \& Couto, C. G. 2015. Medicina interna de pequenos animais. Elsevier Editora, Amsterdan.

Polydoro, A. S., Natalini, C. C., Raiser, A. G. \& Hennemanin, C. R. A. 2006. Analgesia e anestesia epidural em cães e gatos: revisão de literatura. A Hora Veterinária, 25, 31-37.

Rochette, A., Raux, O., Troncin, R., Dadure, C., Verdier, R. \& Capdevila, X. 2004. Clonidine prolongs spinal anesthesia in newborns: a prospective dose-ranging study. Anesthesia \& Analgesia, 98, 56-59.

Schechtmann, G., Wallin, J., Meyerson, B. A. \& Linderoth, B. 2004. Intrathecal clonidine potentiates suppression of tactile hypersensitivity by spinal cord stimulation in a model of neuropathy. Anesthesia \& Analgesia, 99, 135-139.

Silva, J. A. \& Ribeiro-Filho, N. P. 2011. A dor como um problema psicofísico. Revista Dor, $12,138-151$.

Spinosa, H. S., Górniak, S. L. \& Bernardi, M. M. 1999. Farmacologia aplicada à medicina veterinária. Guanabara Koogan, Rio de Janeiro.

Strebel, S., Gurzeler, J. A., Schneider, M. C., Aeschbach, A. \& Kindler, C. H. 2004. Smalldose intrathecal clonidine and isobaric bupivacaine for orthopedic surgery: a doseresponse study. Anesthesia \& Analgesia, 99, 1231-1238.

Valadão, C. A. A., Duque, J. C. \& Farias, A. 2002a. Administração epidural de opióides em cães. Ciência Rural, 32, 347-355.

Valadão, C. A. A., Mazzei, F. \& Oleskovicz, N. 2002b. Avaliação dos efeitos analgésicos da morfina ou cetamina em cães, após injeção epidural, através dos filamentos de von Frey. Arquivo Brasileiro de Medicina Veterinária e Zootecnia, 54, 383-389.

Weissman, C. 1990. The metabolic response to stress: an overview and update. Anesthesiology, 73, 308-327.

Article History:

Received 5 July 2017

Accepted 1 August 2017

Available on line 21 August 2017

License information: This is an open-access article distributed under the terms of the Creative Commons Attribution License 4.0, which permits unrestricted use, distribution, and reproduction in any medium, provided the original work is properly cited. 